\title{
Conventional versus Ultrasound-Guided Transbronchial Needle Aspiration for the Diagnosis of Hilar/Mediastinal Lymph Adenopathies: A Randomized Controlled Trial
}

\author{
Martina Bonifazi $i^{a, b}$ Irene Tramacere ${ }^{c}$ Lina Zuccatosta ${ }^{b}$ Federico Mei ${ }^{b}$ \\ Michele Sediari $^{b}$ Maria Cristina Paonessa ${ }^{d}$ Stefano Gasparini ${ }^{a}$ b

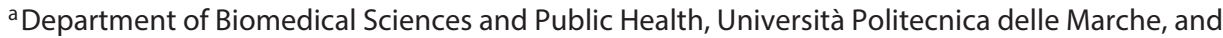 \\ bPulmonary Diseases Unit, Department of Internal Medicine, Azienda Ospedaliero-Universitaria "Ospedali Riuniti", \\ Ancona, 'Scientific Direction, Fondazione IRCCS Istituto Neurologico Carlo Besta, Milan, and dSection of \\ Respiratory Diseases, Department of Medical and Surgical Sciences, University "Magna Graecia" of \\ Catanzaro, Catanzaro, Italy
}

\section{Keywords}

Endobronchial ultrasound-guided transbronchial needle aspiration - Conventional transbronchial needle aspiration . Hilar/mediastinal lymphadenopathy · Sensitivity ·

Randomized controlled trial

\begin{abstract}
Background: Conventional transbronchial needle aspiration (c-TBNA) and endobronchial ultrasound-guided transbronchial needle aspiration (EBUS-TBNA) are both valuable diagnostic techniques for the diagnosis of hilar/mediastinal lesions. Although a superiority of EBUS-TBNA over c-TBNA may be expected, evidence-based data on a direct comparison between these 2 procedures are still lacking. Objectives: We aimed to test the superiority of EBUS-TBNA over c-TBNA in a randomized trial and to evaluate the cost-effectiveness profile of a staged strategy, including C-TBNA as initial test followed by EBUS-TBNA, in case of inconclusive results at rapid on-site evaluation. Methods: Eligible patients were randomized 1:1 to either the EBUS-TBNA or C-TBNA group. The primary endpoint was to test the superiority of EBUS-
\end{abstract}

๑ 2017 S. Karger AG, Basel
TBNA sensitivity over c-TBNA. The secondary endpoints included the sensitivity of the staged strategy, as well as costs and safety related to each procedure and to their sequential combination. Results: A total of 253 patients were randomized to either EBUS-TBNA $(n=127)$ or c-TBNA $(n=126)$, and 31 patients of the c-TBNA group subsequently underwent EBUS-TBNA. The sensitivity of EBUS-TBNA was higher, but not significantly superior to that of c-TBNA (respectively. 92\% [95\% Cl 87-97] and 82\% [95\% Cl 75-90], $p>0.05$ ). The sensitivity of the staged strategy was $94 \%$ (95\% Cl 89-98). No major adverse events occurred. Conclusions: EBUS-TBNA was the single best diagnostic tool, although not significantly superior to C-TBNA. Due to the favorable cost-effectiveness profile of their sequential combination, in selected scenarios with a high probability of success from the standard procedure, these should not be necessarily intended as competitive and the staged strategy could be considered in clinical practice.

(c) 2017 S. Karger AG, Basel

This study was registered at www.clinicaltrials.gov (NCT01658280).

\section{KARGER}

E-Mail karger@karger.com

www.karger.com/res
Stefano Gasparini, MD

Pulmonary Diseases Unit, Department of Internal Medicine

Azienda Ospedaliero-Universitaria "Ospedali Riuniti",

Via Conca 71, IT-60120 Ancona (Italy)

E-Mail s.gasparini@fastnet.it 


\section{Introduction}

The last decades have witnessed a huge revolution of the role of interventional pulmonology in diagnostic workup and management of thoracic diseases. The advent of transbronchial aspiration techniques represents one of the most important advances in this context, as it has extended pulmonologists' perspective beyond the airways, leading to the diagnosis of mediastinal lesions as well as staging of cancers, avoiding additional or more invasive interventions [1].

Conventional transbronchial needle aspiration (cTBNA) via flexible bronchoscopy came first into light in the late 1970s [2]. Since then, several studies worldwide have confirmed its valuable cost-effectiveness and safety profile, in particular for the diagnosis of hilar/mediastinal lymph adenopathies. However, the diagnostic yield of cTBNA seems to be related to both clinical and procedural factors, including lymph node size and location, the underlying type of disease, and the operator's skills [3]. Metaanalyses of the published literature documented, indeed, a highly variable sensitivity, ranging from 14 to $100 \%$, with different pooled estimates according to the prevalence of mediastinal diseases, while specificity was overall excellent, as false positives are nearly anecdotal [3-5].

More recently, advances in technology have led to the development of endobronchial ultrasound-guided transbronchial needle aspiration (EBUS-TBNA) [6]. The echoendoscope offers the operator a real-time visualization of lymph nodes and surrounding structures, enabling to direct the tip of the needle to the target area. Overall, the pooled sensitivity of EBUS-TBNA for the diagnosis of hilar/mediastinal lesions was over $90 \%$ in patients with suspected/known lung cancer and around $80 \%$ in those with sarcoidosis [7-9].

Although a superiority of the image-guided over the conventional procedure could be reasonably supposed by comparisons of pooled estimates from the literature, evidence-based data from properly designed randomized studies are still lacking. To date, 3 randomized investigations limited to patients with sarcoidosis only and including other techniques have been actually performed [1012].

Moreover, in daily practice, because of the higher costs, as well as timing and specific skills related to the ultrasound technology, EBUS-TBNA is not available in several institutions. Even where both the techniques are used, the choice between them seems to be based more on empirical evaluations of operators rather than on established diagnostic algorithms.

Conventional TBNA vs. EBUS-TBNA
Therefore, the main purpose of the present study was, primarily, to test the superiority of EBUS-TBNA over cTBNA for the diagnosis of hilar/mediastinal lesions in a randomized controlled trial. We aimed also to evaluate the cost-effectiveness profile of a staged strategy, according to which patients initially randomized to c-TBNA underwent a subsequent shift to EBUS-TBNA in case of inadequate c-TBNA sampling at rapid on-site evaluation (ROSE) [13] in the same bronchoscopy session. Secondary endpoints included evaluation of the sensitivity of this staged strategy, as well as safety profile, procedural time, and costs related to each procedure separately and to their sequential combination.

\section{Materials and Methods}

This was a single-center, prospective, randomized controlled trial conducted at the Pulmonary Disease Unit of the Azienda Ospedaliero-Universitaria "Ospedali Riuniti" (Ancona, Italy) between August 2012 and November 2015 (Clinical trial.gov No. NCT01658280). The study protocol was approved by the Institutional Ethics Committee of the Azienda Ospedaliero-Universitaria Ospedali Riuniti (approval No. 209457), and written informed consent was obtained from all participants.

Patient Selection and Study Procedures

Consecutive patients aged $\geq 18$ years and with at least 1 hilar/ mediastinal lymph adenopathy $>1 \mathrm{~cm}$ (short axis) on computed tomography (CT) scan in at least 1 lymph nodal station (except $2 \mathrm{R}$ and $2 \mathrm{~L}$ ) were eligible for inclusion, in absence of the following exclusion criteria: (1) severe refractory hypoxemia; (2) uncontrolled coagulopathy; (3) unstable hemodynamic status; and (4) failure to provide an informed consent.

Eligible patients were randomized 1:1 by a computer-generated random-allocation system to either the EBUS-TBNA or cTBNA group. In the latter group, patients with inadequate results at ROSE subsequently underwent EBUS-TBNA in the same bronchoscopy session. An experienced operator (S.G.) performed all bronchoscopic procedures in a dedicated suite with local anesthesia (lidocaine 2\%) under conscious sedation (midazolam $2.5 \mathrm{mg}$ i.v., phentanyl $0.5 \mathrm{mg}$ i.v.), using either a flexible bronchoscope (Olympus BF-IT160, Olympus BF-IT180) with a retractable 19-gauge needle (ConMed MW-319 or Olympus NA601D-1591) or an echobronchoscope (Olympus BF-UC180F) with a 22-gauge needle (Olympus NA-201SX-4022) according to allocation. Three needle passes per each lymphadenopathy/lesion were obtained. The material was firstly smeared on clean glass slides: one smear was immediately stained by a rapid method (Hemacolor; Merck \& Co, Inc.) for ROSE, and the other slides were wet fixed in 95\% ethanol and processed later for definitive cytologic evaluation. The ROSE was performed in a blind fashion by an experienced cytopathologist, who real-time evaluated the adequacy of samples for diagnosis. The specimen was considered as adequate if it presented enough cells to diagnose a specific benign/ malignant condition, or a preponderance of lymphocytes, and as inadequate in case of a preponderance of bronchial cells, a minor-

Respiration 2017;94:216-223 217 


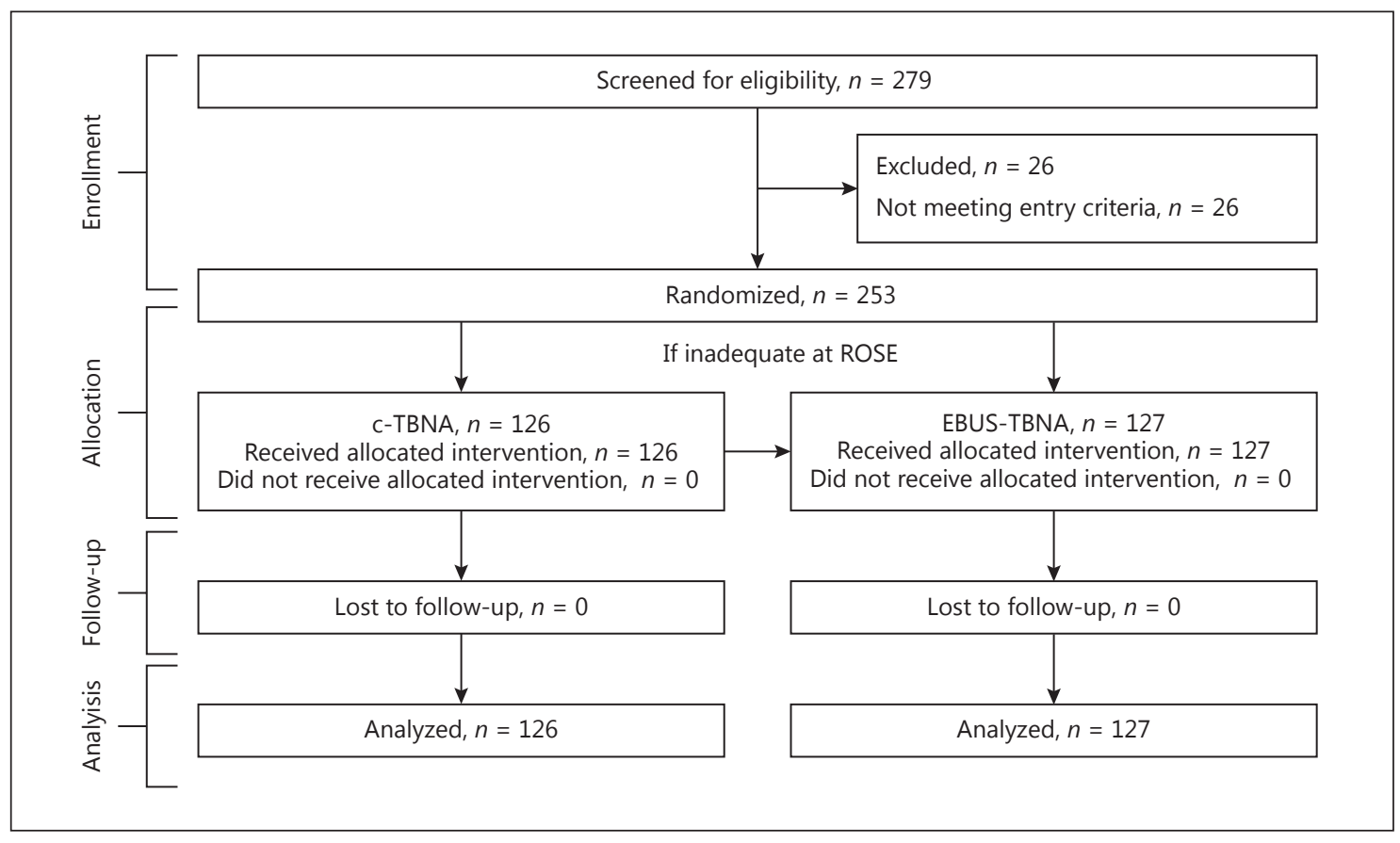

Fig. 1. The Consolidated Standards of Reporting Trials flow diagram. c-TBNA, conventional transbronchial needle aspiration; EBUS-TBNA, endobronchial ultrasound-guided transbronchial needle aspiration; ROSE, rapid on-site evaluation.

ity or no lymphocytes, no features specific to a diagnosis, and in case of doubt.

Then, the material obtained from the remaining passes was fixed in formalin and sent for definitive histological diagnosis.

Clinical history, baseline patient characteristics, lymph node size (short axis) and location, number of lymph nodes sampled, complications, procedural time (from the insertion to the removal of the scope), and final cytological and histological diagnoses were recorded and reported on case report forms. When more than one approachable lymph adenopathy/lesion was present, only samples obtained from the bigger one, were considered for the analysis of primary outcome.

\section{Endpoints and Statistical Analysis}

The primary endpoint was to test the superiority of EBUSTBNA sensitivity over c-TBNA sensitivity. Moreover, a subgroup analysis was performed to evaluate the role of lymph node size $(<2$ vs. $\geq 2 \mathrm{~cm}$ ) and location on the main outcome.

The secondary endpoints included the sensitivity of the staged strategy (c-TBNA and subsequent EBUS-TBNA in case of inadequate sampling at ROSE), procedural time, costs, and safety profile related to each procedure separately and to their sequential combination. Costs of each study arm were derived from the national price of needles employed, without taking into account costs related to the initial purchase and subsequent maintenance of the single instrument and technology.

The primary endpoint was assessed according to intention-totreat analysis. A sample size of at least 252 patients was calculated in order to detect a significant difference of $14 \%$ in sensitivity between the c-TBNA and EBUS-TBNA arms (power: $1-\beta=0.80$; significance level: $\alpha=0.05)$. The sensitivity was established according to definitive cytological and/or histological assessment for each procedure separately (regardless of shift in case of c-TBNA arm as samples were sent for the final evaluation even if patients subsequently underwent EBUS-TBNA) and for the staged strategy (including the sensitivity of TBNA in "non-shifted" patients and the sensitivity of EBUS-TBNA in "shifted" subjects). A case was considered as positive when it was possible to recognize a specific disease, while false negative cases were defined as patients subsequently diagnosed with malignant or benign conditions at later investigations (i.e., thoracic surgery, exam repetition) or during follow-up.

Descriptive statistics were provided for demographic and clinical characteristics. Quantitative variables were summarized by means with standard deviation (SDs), or by relative frequencies, as appropriate. Comparisons were performed with the Student $t$ test (or the Mann-Whitney test for skewed distributions) [14] and with $\chi^{2}$ or Fisher exact test, as appropriate. Student $t$ test (or MannWhitney test) was used to compare continuous variable, and Pearson $\chi^{2}$ test (or Fisher exact test) for categorical variables. Sensitivity values were provided with the corresponding $95 \%$ confidence interval $(95 \% \mathrm{CI})$.

\section{Results}

Over the study period (January 2013 to July 2015), out of 279 consecutive patients screened for eligibility, 253 patients were randomized to either the c-TBNA or EBUS- 
Table 1. Baseline characteristics of the study population

\begin{tabular}{|c|c|c|c|c|}
\hline & $\begin{array}{l}\text { Total } \\
(n=253)\end{array}$ & $\begin{array}{l}\text { c-TBNA } \\
(n=126)\end{array}$ & $\begin{array}{l}\text { EBUS-TBNA } \\
(n=127)\end{array}$ & $p$ value \\
\hline Mean age $\pm S D$, years & $64.9 \pm 12.2$ & $64.1 \pm 12.6$ & $65.6 \pm 11.8$ & 0.35 \\
\hline Male sex & $178(70.3)$ & $87(69.0)$ & $91(71.6)$ & 0.65 \\
\hline Mean lymph node size \pm SD & $2.63 \pm 1.4$ & $2.63 \pm 1.4$ & $2.64 \pm 1.5$ & 0.90 \\
\hline$<2 \mathrm{~cm}$ & 85 & 41 & 44 & \\
\hline$\geq 2 \mathrm{~cm}$ & 168 & 85 & 83 & \\
\hline Location of lymph nodes sampled & & & & 0.31 \\
\hline 7 & $95(37.5)$ & $43(34.1)$ & $52(40.9)$ & \\
\hline $4 \mathrm{R}$ & $50(19.8)$ & $28(22.2)$ & $22(17.3)$ & \\
\hline $4 \mathrm{~L}$ & $31(12.3)$ & $16(12.7)$ & $15(11.8)$ & \\
\hline $10 \mathrm{R}$ & $18(7.1)$ & $8(6.3)$ & $10(7.9)$ & \\
\hline $10 \mathrm{~L}$ & $16(6.3)$ & $9(7.2)$ & $7(5.5)$ & \\
\hline $11 \mathrm{R}$ & $23(9.1)$ & $13(10.3)$ & $10(7.9)$ & \\
\hline $11 \mathrm{~L}$ & $20(7.9)$ & $9(7.2)$ & $11(8.7)$ & \\
\hline
\end{tabular}

Values are numbers with percentages in parentheses, except where otherwise indicated. SD, standard deviation; c-TBNA, conventional transbronchial needle aspiration; EBUS-TBNA, endobronchial ultrasound-guided transbronchial needle aspiration.

Table 2. Sensitivity of c-TBNA, EBUS-TBNA, and staged strategy according to lymph node stations

\begin{tabular}{llll}
\hline $\begin{array}{l}\text { Location of lymph } \\
\text { nodes sampled }\end{array}$ & $\begin{array}{l}\text { c-TBNA } \\
(n=126)\end{array}$ & $\begin{array}{l}\text { EBUS-TBNA } \\
(n=127)\end{array}$ & $\begin{array}{l}\text { Staged } \\
\text { strategy }\end{array}$ \\
\hline $7(n=95)$ & 84 & 92 & 95 \\
$4 \mathrm{R}(n=50)$ & 80 & 93 & 93 \\
$4 \mathrm{~L}(n=31)$ & 66 & 83 & 74 \\
10R $(n=18)$ & 86 & 83 & 76 \\
10L $(n=16)$ & 88 & 100 & 96 \\
$11 \mathrm{R}(n=23)$ & 81 & 91 & 89 \\
$11 \mathrm{~L}(n=20)$ & 66 & 82 & 82 \\
\hline Total, \% & $82(95 \% \mathrm{CI}$ & $92(95 \% \mathrm{CI}$ & $94(95 \% \mathrm{CI}$ \\
& $75-90)$ & $87-97)$ & $89-98)$ \\
\hline
\end{tabular}

CI, confidence interval; c-TBNA, conventional transbronchial needle aspiration; EBUS-TBNA, endobronchial ultrasoundguided transbronchial needle aspiration.

TBNA group (Fig. 1). The main baseline characteristics of the study population are summarized in Table 1. Overall, the mean age was $64.9( \pm 12.2)$ years, the majority of patients were males $(70 \%)$, the mean lymph node short-axis diameter was $2.63 \mathrm{~cm}( \pm 1.4)$, and the most frequently lymph node stations sampled were 7 and $4 \mathrm{R}$ (respectively, 37.5 and $19.8 \%$ ). There were no significant differences in baseline characteristics between the study arms. The final diagnosis was malignancy in 189 patients (adenocarcino- ma, $n=94$; squamous cell carcinoma, $n=22$; small cell carcinoma, $n=27$; nonsquamous cell carcinoma-NSCLC, $n=4$; metastasis from extrathoracic tumors, $n=26$; neuroendocrine large cell carcinoma, $n=3$; and lymphoma, $n=13$ ) and benign condition in the remaining 64 patients (reactive lymphadenopathy, $n=34$; sarcoidosis, $n=27$; Mycobacterium avium complex infection, $n=2$; and amyloidosis, $n=1)$. A complete genotyping of adenocarcinoma (EGFR, ALK, and KRAS testing) as well as immmunohistochemical studies were obtained for all cases, except in 4 ( 1 in the EBUS-TBNA arm and 3 in the c-TBNA arm).

Overall, the sensitivity of c-TBNA and EBUS-TBNA was respectively $82 \%$ (95\% CI 75-90) and 92\% (95\% CI 87-97). Although the EBUS-TBNA sensitivity was higher than that of c-TBNA, this was not significantly different $(p>0.05)$. Of 126 patients randomized to c-TBNA, 31 (24.6\%) subsequently underwent EBUS-TBNA, as the materials obtained through the conventional procedure were considered as inadequate at ROSE, and the final sensitivity of this staged strategy was $94 \%$ (95\% CI 89-98) (Fig. 2). The subgroup analysis did not show a significant difference in sensitivity values according to the main lymph node stations sampled. In particular, the sensitivity of c-TBNA, EBUS-TBNA, and the staged strategy were, respectively, 84,92 , and $95 \%$ for station 7 , and 80 , 93, and $93 \%$ for station $4 \mathrm{R}$ (Table 2). Data from the remaining stations, although limited to a small sample size, are presented in Table 2. Sensitivity values of c-TBNA, 


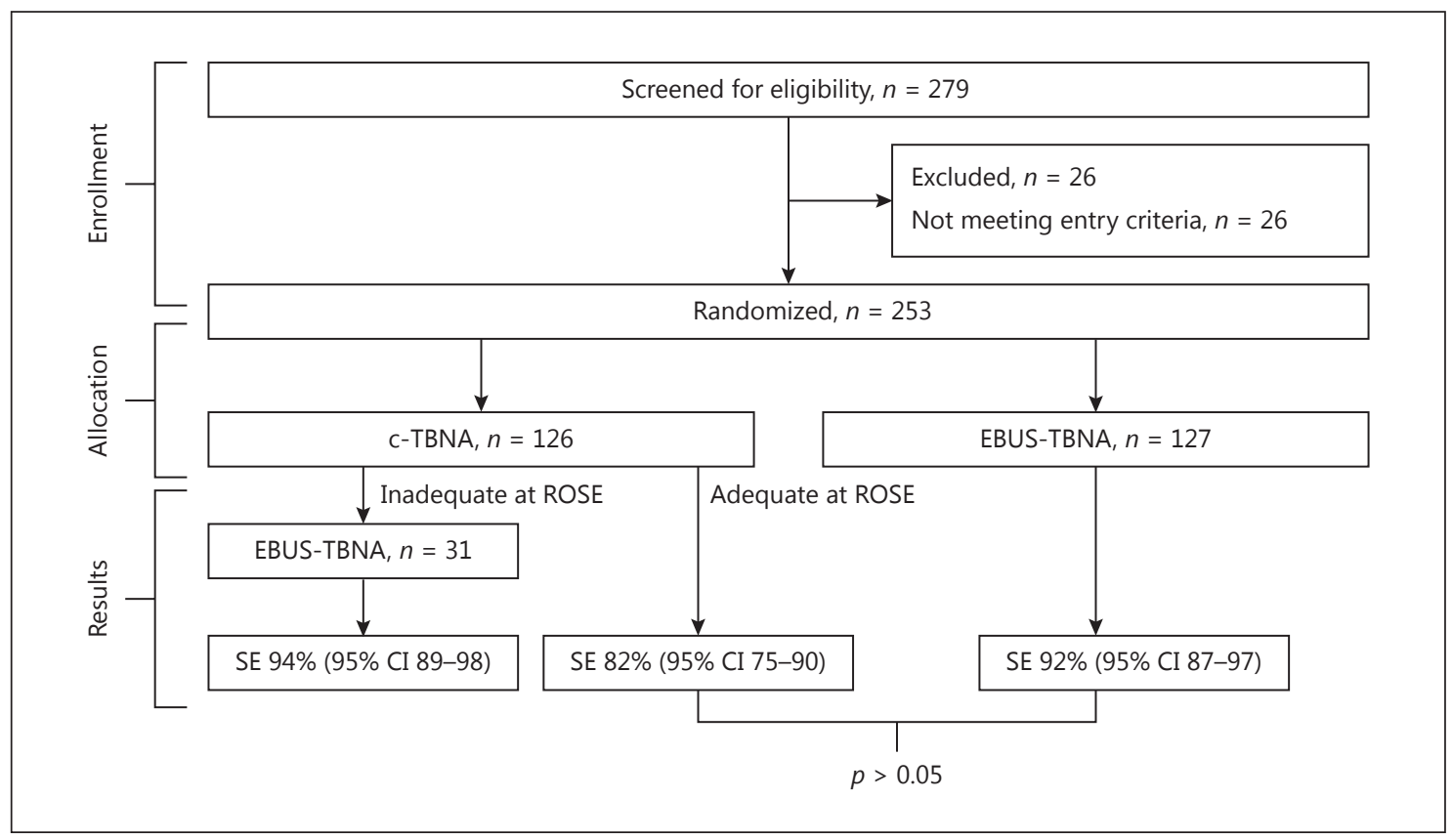

Fig. 2. Sensitivity of c-TBNA, EBUS-TBNA, and staged strategy. c-TBNA, conventional transbronchial needle aspiration; CI, confidence interval; EBUS-TBNA, endobronchial ultrasound-guided transbronchial needle aspiration; ROSE, rapid on-site evaluation; SE, sensitivity.

EBUS-TBNA, and the staged strategy according to lymph node size were respectively $63 \%, 88 \%(p=0.10)$, and $77 \%$ $(p=0.45)$ for lesions $<2 \mathrm{~cm}$, and $88 \%, 93 \%(p=0.27)$, and 93\% ( $p=1$ ) for lesions $\geq 2 \mathrm{~cm}$ (data not shown). The results of c-TBNA, EBUS-TBNA, and the staged strategy for lesions $\leq 1.5 \mathrm{~cm}(n=31)$ were, respectively, 59, 85 , and $68 \%$ (data not shown).

The mean procedural time of c-TBNA, EBUS-TBNA, and the staged strategy were respectively 15,20 , and 34 min. The cost of the c-TBNA arm (126 procedures, EUR 102 each needle) was EUR 12.852, the cost of the EBUSTBNA arm (127 procedures, EUR 152 each needle) was EUR 19.310, and that of the staged strategy was EUR 17.564 (126 c-TBNA needle plus 31 EBUS-TBNA needle).

No major complications occurred. Minor complications included one mild, self-limiting bleeding in the cTBNA arm and one transient laryngospasm in the EBUSTBNA arm.

\section{Discussion}

To our knowledge, the present study is the first randomized trial on a direct comparison between c-TBNA and linear EBUS-TBNA for the diagnosis of hilar/medi- astinal lesions. It showed that the sensitivity of the imageassisted procedure was higher than that of the conventional technique, but not significantly different. Our findings also suggest that a staged strategy, with c-TBNA as first diagnostic step, followed by EBUS-TBNA in case of inconclusive results at ROSE, may present the best costeffectiveness profile, without increasing complications. In fact, less than one quarter $(24.6 \%)$ of patients who initially underwent c-TBNA actually required a shift to EBUS-TBNA, and the final cost of such algorithm was lower than that of EBUS-TBNA alone. Such a saving, although slight, may be significant in some institutions worldwide with limited resources. Moreover, the different costs related to purchase and maintenance of the whole equipment, not included in the present evaluation, should also be considered for the overall estimation of expenses. On the other hand, the sequential combination would require a longer procedural time for subjects undergoing both the procedures, and it could result in hypothetical higher risks. However, the complication rate related to these diagnostic techniques is almost negligible, as serious adverse events are extremely rare, as reported in the literature [15] and confirmed in our investigation. The almost 2-fold longer mean procedural time of the staged strategy as compared to that of EBUS-TBNA and 
c-TBNA as single techniques (respectively, 34, 20, and 15 $\min$ ) should be considered. However, the overall procedural time of the diagnostic algorithm, considering that only a minority of patients undergoing both procedures, was not substantially different from that of the EBUSTBNA arm (respectively, 2,510 and 2,543 $\mathrm{min}$ ).

It is worth underlying that the role of EBUS-TBNA remains unquestionably incomparable to that of $\mathrm{c}$ TBNA, especially in diagnostic workup of smaller lesions and for mediastinal cancer staging. The lymph node size seems, indeed, to highly influence the success of aspiration techniques. However, in the present study, the sensitivity of c-TBNA and EBUS-TBNA for lymph nodes $<2$ $\mathrm{cm}$ (63 vs. $88 \%$ ) was hugely different, further supporting the supremacy of the image-assisted procedure in this context. The irreplaceable role of EBUS-TBNA is even more straightforward in the context of mediastinal cancer staging, as it also allows to detect nodes not visible on CT, increasing the accuracy of the evaluation. Based on recent data, in patients with a central tumor or N1 node involvement, a pathological confirmation of nodal staging is recommended despite the absence of mediastinal node enlargement on imaging, as the false negative rate of CT and PET scanning in these cases is approximately $20-25 \%$ $[16,17]$. When combined with the esophageal endoscopic ultrasound-guided technique (EUS-FNA) in a complementary approach, as recommended in latest international guidelines, almost all mediastinal nodes can be virtually accessed. Therefore, the combined EBUS/EUS approach is the only recommended mini-invasive strategy in this context.

Interestingly, no previous prospective studies have ever compared c-TBNA and linear EBUS-TBNA in unselected populations with lymph adenopathies/masses of unknown origin, but in patients with suspected sarcoidosis only. In this context, although the sensitivity of EBUS-TBNA seemed to be individually higher than that of c-TBNA, the diagnostic yield of these 2 techniques, when combined with endobronchial biopsy and transbronchial lung biopsy, was not significantly different [10]. The only RCTs available for the diagnosis of enlarged lymph nodes/masses in unselected population have compared the diagnostic yield of c-TBNA and EBUS-guided TBNA, which is completely different from EBUS-TBNA. This consisted in a standard TBNA preceded by the insertion of a radial US probe into the working channel of the flexible scope to visualize the target lesion, subsequently removed to allow the transbronchial sampling [18]. In the study by Shannon et al. [19], the diagnostic accuracy of EBUS-guided TBNA and c-TBNA

Conventional TBNA vs. EBUS-TBNA was not significantly different, while in the 2 latest investigations, a superiority of the image-assisted procedure was documented only for stations other than subcarinal $[18,20]$.

Further methodological differences between the present study and previous investigations included the use of a 19-gauge needle for every patient in the c-TBNA arm, rather than the 21/22-gauge, and the use of ROSE, that allowed to test the staged strategy as well. Although recent randomized studies failed to detect a significant improvement in the diagnostic yield of transbronchial aspiration techniques with the addition of ROSE [21], this has been shown to reduce the number of needle passes, the complications rate, and the need for additional procedures by increasing the success rate of molecular genotyping [22]. In the era of targeted lung cancer therapy, indeed, a sample should be considered as diagnostic only if it provides an amount of cells suitable for both tumor subtyping and molecular genotyping [23]. Of note, in the present study, a complete molecular characterization was, in fact, obtained in more than $95 \%$ of patients with NSCLC, regardless of the technique employed. Notwithstanding, ROSE is still not a widespread procedure as, in most interventional pulmonology centers, due to the lack of time and resources, on-site cytopathologists are not routinely available [24]. This major limitation has to be taken into account when considering the feasibility of the staged strategy in clinical practice, as the every-day availability of a ROSE service requires additional costs, mostly related to the need of dedicated personnel source. However, we have recently demonstrated that a bronchoscopist, after a short intensive educational training in pulmonary cytopathology, may be able to on-site assess the adequacy of specimens by him/herself, overcoming, thus, issues related to the routinely involvement of cytopathologists and further reducing costs [25]. In the current RCT, ROSE was performed by a cytopathologist, rather than by a pulmonologist, only because such data were not yet available when the study was conceived. Major methodological strengths included the prospective randomized design, the consecutive enrollment of patients, leading to the inclusion of different diseases, and the large sample size.

This study presents a number of limitations. First, the reproducibility of our findings worldwide may be affected by the fact that all procedures were performed in a single center by a single bronchoscopist with a long-life experience with c-TBNA (S.G.). The sensitivity of the standard procedure was, indeed, higher than that expected from meta-analyses of the literature $[4,5]$. However, in a recent

Respiration 2017;94:216-223 DOI: $10.1159 / 000475843$ 
systematic review of studies on the accuracy of c-TBNA, a number of clinical and procedural aspects have been identified as predictors of a successful aspirate for the diagnosis of mediastinal lymphadenopathies/masses, including a lymph node size $>2 \mathrm{~cm}$, station 7 and $4 \mathrm{R}$ as site of samples, an underlying malignant condition, and the use of a histological needle by an "experienced" bronchoscopist to provide both histological and cytological samples [3]. Most of these factors have been actually verified in the present study, partly explaining such a satisfying c-TBNA sensitivity. In particular, approximately two thirds of the study population presented with lymph nodes $\geq 2 \mathrm{~cm}$, and the results according to lymph node size confirmed the higher sensitivity of c-TBNA in this subgroup (88\%) compared to that obtained from patients with smaller lymph nodes $(63 \%)$. If the majority of patients had presented with smaller lesions in stations other than 7 and $4 \mathrm{R}$ and a prevalence of benign conditions [26], the sensitivity obtained would have been maybe lower. However, the purpose of the current study, although conducted in a randomized fashion according to a predefined protocol, was to reflect as much as possible a real-world scenario, and thus, patients were consecutively enrolled without restrictions exactly as they usually present in clinical practice.

The different size of needles used for c-TBNA (19gauge) and EBUS-TBNA (22-gauge) should be also taken into account in the interpretation of results. Nevertheless, the 19-gauge needle for EBUS-TBNA has become available only recently on the market, and no data yet exist on a direct comparison between the sensitivity from different needle sizes.

A further limitation is represented by the lack of a standardized verification of results by surgical procedures. Positive results were generally assumed to be true positives and were not surgically confirmed, avoiding useless invasive interventions [4]. Therefore, the specificity from both these procedures has not been reported, assuming that it was $100 \%$. Negative results, instead, were all verified, but by different reference tests (i.e., mediastinoscopy, mediastinotomy, thoracotomy, video-assisted thoracoscopy, and median sternotomy), or by clinical followup alone, when surgery was not indicated, leading to a potential verification bias.

The present randomized study, comparing c-TBNA and linear EBUS-TBNA for the diagnosis of hilar/mediastinal lesions of unknown origin, showed that EBUSTBNA was the single best diagnostic tool, although the sensitivity of these techniques did not significantly differ.

This implies that the conventional technique should be still considered as a valuable diagnostic tool, especially in centers with limited resources where EBUS-TBNA is not available. Our results also suggest that c-TBNA and EBUS-TBNA should not be necessarily intended as competitive, as, in selected scenarios with a high probability of success from the standard procedure, they can be included in a staged strategy with c-TBNA as initial test, followed by EBUS-TBNA, in case of inconclusive results at ROSE, without increasing complications and reducing costs. In other words, in presence of enlarged hilar/mediastinal lymph nodes, it would be reasonable, in experienced hands, to firstly employ the conventional technique, leaving the imagine-assisted procedure as subsequent step. In the remaining cases, the role of EBUS-TBNA remains unquestionable, especially when a medical mediastinoscopy is required.

\section{Disclosure Statement}

None of the authors has any potential conflicts of interest to disclose.

\section{References}

1 Yang H, Zhang Y, Wang KP, Ma Y: Transbronchial needle aspiration: development history, current status and future perspective. J Thorac Dis 2015;7:S279-S286.

2 Wang KP, Terry P, Marsh B: Bronchoscopic needle aspiration biopsy of paratracheal tumors. Am Rev Respir Dis 1978;118:17-21.

3 Bonifazi M, Zuccatosta L, Trisolini R, Moja L, Gasparini S: Transbronchial needle aspiration: a systematic review on predictors of a successful aspirate. Respiration 2013;86:123134.
4 Holty JE, Kuschner WG, Gould MK: Accuracy of transbronchial needle aspiration for mediastinal staging of non-small cell lung cancer: a meta-analysis. Thorax 2005;60:949955.

5 Toloza EM, Harpole L, Detterbeck F, McCrory DC: Invasive staging of non-small cell lung cancer: a review of the current evidence. Chest 2003;123:157S-166S.

6 Annema JT, Versteegh MI, Veselic M, Voigt P, Rabe KF: Endoscopic ultrasound-guided fine-needle aspiration in the diagnosis and staging of lung cancer and its impact on surgical staging. J Clin Oncol 2005;23:8357-8361.

7 Agarwal R, Srinivasan A, Aggarwal AN, Gupta D: Efficacy and safety of convex probe EBUSTBNA in sarcoidosis: a systematic review and meta-analysis. Respir Med 2012;106:883-892.

8 Chandra S, Nehra M, Agarwal D, Mohan A: Diagnostic accuracy of endobronchial ultrasound-guided transbronchial needle biopsy in mediastinal lymphadenopathy: a systematic review and meta-analysis. Respir Care 2012;57:384-391. 
9 Adams K, Shah PL, Edmonds L, Lim E: Test performance of endobronchial ultrasound and transbronchial needle aspiration biopsy for mediastinal staging in patients with lung cancer: systematic review and meta-analysis. Thorax 2009;64:757-762.

10 Gupta D, Dadhwal DS, Agarwal R, Gupta N, Bal A, Aggarwal AN: Endobronchial ultrasound-guided transbronchial needle aspiration vs. conventional transbronchial needle aspiration in the diagnosis of sarcoidosis. Chest 2014; 146:547-556.

11 Li K, Jiang S: A randomized controlled study of conventional TBNA versus EBUS-TBNA for diagnosis of suspected stage I and II sarcoidosis. Sarcoidosis Vasc Diffuse Lung Dis 2014;31:211-218.

12 Tremblay A, Stather DR, Maceachern P, Khalil M, Field SK: A randomized controlled trial of standard vs. endobronchial ultrasonography-guided transbronchial needle aspiration in patients with suspected sarcoidosis. Chest 2009;136:340-346.

13 Gasparini S. It is time for this 'ROSE' to flower. Respiration 2005;72:129-131.

14 Vickers AJ: Parametric versus non-parametric statistics in the analysis of randomized trials with non-normally distributed data. BMC Med Res Methodol 2005;5:35.
15 Gasparini S, Bonifazi M, Wang KP: Transbronchial needle aspirations vs. percutaneous needle aspirations. J Thorac Dis 2015;7:S300S303.

16 Silvestri GA, Gonzalez AV, Jantz MA, Margolis ML, Gould MK, Tanoue LT, Harris LJ, Detterbeck FC: Methods for staging non-small cell lung cancer: diagnosis and management of lung cancer, ed 3. American College of Chest Physicians evidence-based clinical practice guidelines. Chest 2013;143:e211Se250S.

17 Mehta AC, Cicenia J, Yasufuku K: The chef has a knife... endoscopic ultrasound-guided fine-needle aspiration by a pulmonologist. Chest 2015;147:1201-1203.

18 Herth F, Becker HD, Ernst A: Conventional vs. endobronchial ultrasound-guided transbronchial needle aspiration: a randomized trial. Chest 2004;125:322-325.

19 Shannon JJ, Bude RO, Orens JB, Becker FS, Whyte RI, Rubin JM, Quint LE, Martinez FJ: Endobronchial ultrasound-guided needle aspiration of mediastinal adenopathy. Am J Respir Crit Care Med 1996;153:1424-1430.

20 Arslan Z, Ilgazli A, Bakir M, Yildiz K, Topcu S: Conventional vs. endobronchial ultrasound-guided transbronchial needle aspiration in the diagnosis of mediastinal lymphadenopathies. Tuberk Toraks 2011;59:153157.
21 Trisolini R, Cancellieri A, Tinelli C, Paioli D, Scudeller L, Casadei GP, Parri SF, Livi V, Bondi A, Boaron M, Patelli M: Rapid on-site evaluation of transbronchial aspirates in the diagnosis of hilar and mediastinal adenopathy: a randomized trial. Chest 2011;139:395-401.

22 Trisolini R, Cancellieri A, Tinelli C, de Biase D, Valentini I, Casadei G, Paioli D, Ferrari F, Gordini G, Patelli M, Tallini G: Randomized trial of endobronchial ultrasound-guided transbronchial needle aspiration with and without rapid on-site evaluation for lung cancer genotyping. Chest 2015;148:1430-1437.

23 Zakowski MF, Bibbo M: Lung carcinoma in the era of personalized medicine: the role of cytology. Acta Cytol 2012;56:587-589.

24 Mehta AC, Cicenia J: ROSEs are read. Chest 2014;145:7-9.

25 Bonifazi M, Sediari M, Ferretti M, Poidomani G, Tramacere I, Mei F, Zuccatosta L, Gasparini S: The role of the pulmonologist in rapid on-site cytologic evaluation of transbronchial needle aspiration: a prospective study. Chest 2014;145:60-65.

26 Mehta AC, Almeida FA: Choose wisely: endobronchial ultrasound-guided transbronchial needle aspiration for sarcoidosis. Chest 2014; 146:530-532. 